\title{
Comparative Analysis of Constructive Heuristic Algorithms for Transmission Expansion Planning
}

\author{
Phillipe Vilaça Gomes ${ }^{1}$, João Tomé Saraiva ${ }^{2}$ \\ INESC TEC, Department of Electrical and Computer Engineering, Faculty of Engineering, \\ University of Porto, Porto, Portugal (1phillipe.gomes@fe.up.pt, 2jsaraiva@fe.up.pt)
}

\begin{abstract}
Transmission Expansion Planning (TEP) is a complex optimization problem that has the purpose of determining how the transmission capacity of a network should be enlarged, satisfying the increasing demand. This problem has combinatorial nature and different alternative plans can be designed so that many algorithms can converge towards local optima. This feature drives the development of tools that combine high robustness and low computational effort. This paper presents a comparative analysis and a detailed review of the main Constructive Heuristic Algorithms (CHA) used in the TEP problem. This kind of tools combine low computational effort with reasonable quality solutions and can be associated with other tools to use in a subsequent step in order to improve the final solution. CHAs proved to be very effective and showed good performance as the test results will illustrate.
\end{abstract}

Subject Headings. Energy Transport, Cost Analysis, Electrical Engineering

Author Keywords. Transmission Expansion Planning, Constructive Heuristic Algorithms, Paper Review

\section{Introduction}

The increasing electricity demand drives the Power System Expansion (PSE) so that the load is properly supplied. Three main forms can be highlighted to achieve the PSE:

- Installing new generation facilities closer to the demand centers;

- Building new transmission lines connecting generation plants to demand centers;

- A combination of new and more efficient power stations with new transmission facilities.

However, the first option does not have economic or even physical viability in most cases. On the other hand, the second alternative can also enable the optimal dispatch of power plants. A stronger transmission system, in addition to provide more flexibility to dispatch generation, improves system reliability and reduces the likelihood of having congested branches.

The goal of TEP problem is to identify where and when new transmission lines will be constructed to minimize a predefined objective function while supplying the forecasted demand along an extended future. The objective function to be minimized is usually the investment cost combined with the system's operating costs. However this study can be addressed with more than one objective, that is, in a multi-criteria approach. Other objectives found in the literature on TEP are as follows (da Rocha and Saraiva 2013):

- Alleviate transmission congestion;

- Minimize the risk of investments;

- Increase the reliability of the network; 
- Increase the flexibility of system operation while reducing the network charges;

- Minimize the environmental impacts;

- Allow better voltage level regulation.

However, some characteristics of the TEP problem make it difficult to solve it, becoming a real challenge in the power systems area. Among these features are (de Mendonça, Junior, and Marcato 2014):

- Non convex search space;

- Isolated buses;

- Integer nature of the problem;

- Several investment options.

These aforementioned features correspond to major difficulties in developing tools that combine efficiency and computational effort. Most of the tools can be classified into Classical Optimization, Constructive Heuristics, Metaheuristics and Hybrid Tools. Classical Optimization uses decomposition techniques and generally finds global optimal solutions despite having convergence problems in some cases (Rider, Garcia, and Romero 2008). The use of CHAs implies introducing simplifications to identify feasible and reasonable solutions to complex problems (Romero et al. 2005). Metaheuristics are basically heuristics reinforced by a smart search mechanism (Gomes 2015). Finally, the hybrid tools use combinations of the three groups presented above (Gomes, Phillipe Vilaca ; Saraiva 2015).

The CHAs are easy techniques to be implemented, they have low computational effort and converge to acceptable quality solutions. For this reason they are adequate tools to reduce the search space in problems that have high complexity and combinatorial explosion phenomenon of the search space, such as the TEP problem. However, it is expected that this search space reduction is made so as to keep the main expansion routes, that is, to maintain optimal and sub-optimal solutions and only discarding worse quality solutions. In this context, this paper presents a study of the major CHAs used in the TEP problem and has the main objective of analyzing the quality of the solution presented by each $\mathrm{CHA}$ in order to verify if the optimal solutions reported in the literature are not excluded from the reduced search space.

Regarding the structure of the paper, following this Introduction, Section 2 details the models used in the TEP problem, Section 3 describes the formulation of the main CHAs used in the TEP problem, Section 4 presents the results obtained in CHA simulations and Section 5 presents the enumerates the most relevant conclusions obtained through these simulations.

\section{Transmission Expansion Planning: Mathematical Modeling}

The ideal mathematical model for the TEP problem is the AC model (Bent et al. 2014). However, this model has obstacles to its widespread use, such as convergence problems in islanded systems, which are potentiated with the reactive power flow approach. Therefore, the main models that are often used are based on the AC Model adopting several types of relaxations. Such models are DC Model, the Transportation Model and the Hybrid model as described in the next paragraphs.

\subsection{Model}

The DC model requires that the two Kirchhoff's laws are satisfied as well as the transmission capacity of existing and candidate lines. The mathematical formulation of this model is described by (1) to (7). 


$$
\text { Minimize } v=\sum_{(i, j)} c_{i j} n_{i j}
$$

Subject to:

$$
\begin{gathered}
S . f+g=d \\
f_{i j}-\gamma_{i j}\left(n_{i j}^{0}+n_{i j}\right) \cdot\left(\theta_{i}-\theta_{j}\right)=0 \\
\left|f_{i j}\right| \leq\left(n_{i j}+n_{i j}^{0}\right) \cdot f_{i j} \\
0 \leq g_{i} \leq \overline{g_{i}} \\
0 \leq n_{i j} \leq \overline{n_{i j}} \\
n_{i j} \text { entire, } f_{i j} \text { and } \theta_{j} \text { unbounded }
\end{gathered}
$$

In this formulation $c_{i j}$ is the cost of a circuit in path $i-j, n_{i j}$ are the circuits to be built in path $\mathrm{i}-\mathrm{j}, \mathrm{S}$ is the node-branch incidence transposed matrix of the electrical system, $f$ is the vector of power flows, $g$ is the generation vector, $d$ is the demand vector, $\gamma_{i j}$ is the susceptance of the added circuits in path $\mathrm{i}-\mathrm{j},{\stackrel{o}{n_{i j}}}_{\text {is }}$ is the number of circuits in the base topology from $\mathrm{i}$ to $\mathrm{j}$ and $\theta_{i}$ is the voltage angle at bus $\mathrm{i}$.

Equation (1) of this modeling is the objective function, which represents the investment cost in new transmission lines. Equations $(2,3)$ represent the first and the second Kirchhoff's law, the set of constraints described in (4) represent the circuits transmission capacity, constraints (5) refer to the generation limits and (6) refers to the line limits that are added to each i-j path.

\subsection{Transportation Model}

The transportation model only includes the first Kirchhoff law, therefore ignoring the second Kirchhoff law (relaxation of the problem in Section 2.1). As this modeling is incomplete, the optimal solution found may not be the optimal solution of the complete DC Model or may even be unfeasible. Therefore the Transportation Model is similar to the DC Model removing equations ( 3 ) and the limits on $\theta$.

\subsection{Hybrid Model}

This model was proposed with the aim of reducing the relaxation level that is implicit in the Transportation Model while reducing the complexity of the DC Model. In this model, the first Kirchhoff law should be respected in all buses while the second Kirchhoff law is only only enforced in those paths that already existed in the base configuration.

\section{Constructive Heuristic Algorithms (CHA)}

A CHA basically does the expansion process step by step. In this type of techniques it is performed a sensitivity analysis using a function responsible for assessing the system performance and the expansion cost with respect to the addition of a transmission line. Therefore, in each step a circuit is selected by a sensitive indicator that has the following characteristics:

- Be able to identify the most attractive paths to add transmission lines;

- Be a local character indicator;

- There is no convergence guarantees in global optimum. 
The CHAs used in the TEP follow the steps described below:

1. Check if in a given planning horizon the system will meet the demand properly, that is, without load shedding and congestion on the lines;

2. If at least an unfeasibility is detected, it is promoted the addition of a line in a path that is selected based on the sensitivity indicator that was chosen;

3. Update the system with the addition of this line and verify if the system operates properly. If so, the process has converged to a solution. Otherwise, go back to step 2 .

The main CHAs used to solve the problem TEP problem are as follows:

- Garver;

- Minimum Effort;

- Minimum Load Shedding;

- Villasana - Garver, and

- Marginal Network Levi-Calovic.

\subsection{Garver CHA}

This CHA was proposed by (Garver 1970) and uses the relaxed Transportation Model to solve the TEP problem. The Garver CHA relaxes even more the Transportation Model and accepts continuous solutions to $n_{i j}$ value. It is clear that a solution with fractional transmission lines can't be accepted as a global proposed solution. However, it can be used as a strategy in an attempt to find a good solution to these integer variables (transmission lines) through the sensitivity criterion (8).

$$
f_{i j}^{v}=n_{i j} \cdot \overline{f_{i j}}
$$

Thus, the Garver CHA consists of using the corresponding linear programming problem only as a strategy for finding a good solution for planning purposes and it includes the following steps:

1. Take the base configuration as current configuration;

2. Solve the corresponding linear problem (Transportation Model with continuous $n_{i j}$ variables) for the current configuration;

3. If $n_{i j}=0, \forall i j$ then a good solution (feasible solution) was found.

4. Otherwise proceed to step 3.

5. Use the sensitivity criterion (8) to identify the best path. Add a line in this path and update the system with this addition, that is update the current configuration of the network. Return to step 2.

The main problems with this approach are:

- Some added circuits may become irrelevant with the later addition of other more important circuits. This problem can be solved by adding an extra step in which these irrelevant circuits are removed.

- The sensitivity criterion may become inefficient when the (8) has a very small number as outcome, because it represents where the line will be constructed. This problem can be minimized by choosing a threshold for the addition of more attractive circuit.

\subsection{Minimum Effort CHA}

The Minimum Effort CHA was proposed by (Monticelli et al. 1982) and uses the DC Model to solve the TEP problem. The Minimum Effort CHA relaxes the DC Model because it also 
accepts continuous solutions to the $n_{i j}$ variables as an attempt to find a good solution to these originally integer variables (transmission lines) through the sensitivity criterion (9).

$$
S I_{i j}^{m e}=-\frac{1}{2} \cdot\left(\theta_{i}-\theta_{j}\right)^{2} \cdot \gamma_{i j}
$$

Thus, the Minimum Effort CHA consists of using the corresponding linear programming problem only as a strategy for finding a good solution for planning purposes and its application includes the following steps:

1. Take the base configuration as the current configuration;

2. Solve the corresponding linear problem (DC Model with continuous $n_{i j}$ variables) for the current configuration;

3. If there are overloads on lines, go to step 3;

4. Otherwise a good solution (feasible solution) was found.

5. Use the sensitivity criterion (9) to identify the best path. Add a line in this path and update the current configuration of the system with this addition. Return to step 2.

\subsection{Minimum Load Shedding $\mathrm{CHA}$}

The minimum load shedding CHA was proposed by (Pereira and Pinto 1985) and uses a modified DC Model by including fictitious generators $\left(r_{i}\right)$ according to the formulation (10) to (15).

$$
\text { Minimize } w=\sum r_{i}
$$

Subject to:

$$
\begin{gathered}
\text { B. } \theta+g+r=d \\
\left|\theta_{i}-\theta_{j}\right| \leq \bar{\phi}_{i j} \\
0 \leq g_{i} \leq \bar{g}_{i} \\
0 \leq r \leq d \\
\theta_{j} \text { unbounded }
\end{gathered}
$$

In the Minimum Load Shedding $\mathrm{CHA}$, the flows in the lines must be less than the maximum allowed (unlike the Minimum Effort $\mathrm{CHA}$ ), i.e, they must remain between the operating limits. The operating system problems are repaired by fictitious generators, thus carrying out load shedding in the system. Therefore, at each iteration, this CHA minimizes the system load shedding and the process terminates when sufficient circuits capable of eliminating this load shedding are added to the power system. The sensitivity criterion is obtained by expression (16). In this expression $\pi$ is the Lagrange multiplier of constraint (11).

$$
S I_{i j}^{m c}=\frac{\partial Z}{\partial \gamma_{i j}}=-\left(\theta_{i}-\theta_{j}\right) \cdot\left(\pi_{i}-\pi_{j}\right)
$$

Thus, the Minimum Load Shedding CHA consists of performing the following steps:

1. Take the base configuration as the current configuration; 
2. Solve the corresponding linear problem (DC Model (10) to (15)) for the current configuration;

a. If there is Load Shedding in at least one bus, go to step 3;

b. Otherwise a good solution (feasible solution) was found.

3. Use the sensitivity criterion (16) to identify the best path. Add a line in this path and update the current configuration of the system with this addition. Return to step 2.

The application of this CHA can be complicated in cases in which the current configuration is entirely connected. In these cases, a fictitious network should be used to solve this problem. Thus it is defined a fictitious network consisting of fictitious circuits with very small amounts of $n_{i j}$ but with a transmission capacity exceeding a normal circuit.

\subsection{Villasana-Garver CHA}

The Villasana-Garver CHA was proposed by (Villasana, Garver, and Salon 1985) and uses a Hybrid Model to solve the TEP problem. In this CHA it is used a mathematical model that represents two overlapping grids, one that corresponds to the existing circuits in the current configuration and other artificial network composed of existing fictional circuits in all candidate paths. The main idea of this approach is to solve the system operating problems considering only the network with the circuits of the current configuration and use the fictitious network only in the event that the existing circuits in the current configuration are insufficient to solve those problems. The mathematical modeling with two overlapping networks is given by (17) to (23). In this formulation $\Omega_{1}$ represents the set of existing lines in the current configuration, and $\Omega_{2}$ is the set that includes the fictional network candidate paths.

$$
\text { Minimize } v 2=\sum c_{i j} \cdot n_{i j}
$$

Subject to:

$$
\begin{gathered}
S . f+B_{1} \cdot \theta_{1}+g=0 \\
\left|\theta_{i}-\theta_{j}\right| \leq \bar{\phi}_{i j}, \forall(i, j) \in \Omega_{1} \\
\left|f_{i j}\right| \leq \bar{f}_{i j} \cdot n_{i j}, \forall(i, j) \in \Omega_{2} \\
0 \leq g_{i} \leq \overline{g_{i}} \\
n_{i j} \geq 0 \\
f_{i j} \text { and } \theta_{j} \text { unbounded }
\end{gathered}
$$

The CHA can be summarized in the following steps:

1. Take the base configuration as the current configuration;

2. Solve the corresponding linear problem (Hybrid Model (17) to (23)) for the current configuration.

a. If $v 2=0$, that is, $n=0$, then stop because a good feasible configuration has been found.

b. Otherwise calculate the current investment and go to step 3. 
3. Add in the current configuration a new circuit in the fictitious path that leads to the larger power flow among all the fictitious paths.

\subsection{Marginal Network Levi-Calovic}

The Marginal Network Levi-Calovic was proposed by (Levi, V.A. and Calovic, M.S. 1991) and also uses a Hybrid Model to solve the TEP problem. In this CHA, instead of using two overlapping networks such as in the Villasana-Garver CHA, it is carried out the assembly and solution of two mathematical models corresponding to two different networks in each iteration of the algorithm. The first mathematical model is the DC model (24) to (29) for the current configuration. If the system does not operate properly for the current configuration, the algorithm proceeds to the assembly of the marginal network using the results obtained by solving the first mathematical model.

$$
\text { Minimize } w=\sum r_{i}
$$

Subject to:

$$
\begin{gathered}
B_{1} \cdot \theta_{1}+g+r=d \\
\left|\theta_{i}-\theta_{j}\right| \leq \bar{\phi}_{i j}, \forall(i, j) \in \Omega_{1} \\
0 \leq g_{i} \leq \bar{g}_{i} \\
0 \leq r \leq d \\
\theta_{j} \text { unbounded }
\end{gathered}
$$

If the linear problem above does not present load shedding, i.e, if $w=0$, then it was found a feasible solution to the TEP problem. Otherwise it should be added the most attractive circuit to the new current configuration. The most attractive circuit is found from the solution of marginal network which must be built using the problem (24) to (29) taking into account the following aspects:

- Generations in the marginal network are formed by the residual generations, which correspond to the difference between the maximum generation capacity and generation obtained from (24) to (29);

- Demands or marginal network loads are formed by the load shedding values that are obtained after solving (24) to (29).

- The topology of the marginal network has two types of circuits as follows:

- Saturated Circuits: If current circuits in the path $i-j$ are at the limit in the solution of problem (24) to (29), the corresponding circuit in the marginal network must have a cost $c_{i j}$. In this case, there is no residual capacity in this way to be profitable because the circuits are working on the limit;

- Unsaturated circuits: If current circuits in the path $i-j$ are operating below their limits, the corresponding circuits in the marginal network must have a cost equal to zero by the existing residual capacity and cost $c_{i j}$ above this residual capacity.

So, considering these indications, the marginal network originates the mathematical model (30) to (35). 


$$
\text { Minimize } v 1=\sum c_{i j} \cdot n_{i j}
$$

Subject to:

$$
\begin{gathered}
\text { S. } f+g_{m}=d_{m} \\
\left|f_{i j}\right| \leq \bar{f}_{i j} \cdot n_{i j} \\
0 \leq g_{m} \leq \overline{g_{m}} \\
n_{i j} \geq 0 \\
f_{i j} \text { unbounded }
\end{gathered}
$$

In this formulation $g_{m}$ and $d_{m}$ are the demand and generation residual vectors respectively, $c_{i j}$ is the cost and it assumes the form given in Figure 1 . In this Figure $\overline{n^{\prime}{ }_{i j}}$ is the circuit available capacity that is possible to be used at zero cost in the marginal network.

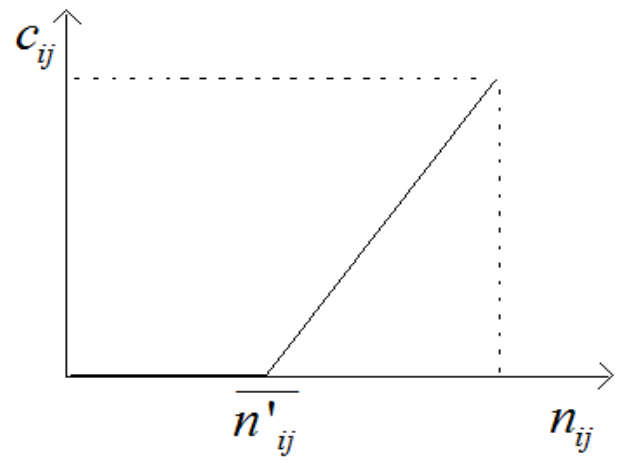

Figure 1: Cost function in marginal network

\section{Results}

The CHA techniques presented in the previous chapter were simulated using the Garver 6Bus academic system with and without generation rescheduling. Not considering rescheduling means that the generation pattern is pre-determined in order to supply the demand while if rescheduling is considering then generation outputs can be change in order to minimize the overall cost. It is then clear that admitting rescheduling gives the problem extra flexibility and so the expansion cost will be smaller when compared with the one that is obtained without rescheduling. The algorithms was implemented in MATLAB, running on an Intel i3, 2.53GHz, 4GB RAM, hardware platform.

The Garver system consists of 6 bars, 6 existing circuits at the base topology, 15 candidate circuits for expansion (it is allowed to build up to 4 circuits in each path) and the demand is $760 \mathrm{MW}$. The optimal solutions for this system have a cost of 110 million dollars (additions in $n_{3,5}, n_{4,6}, n_{4,6}$ and $n_{4,6}$ branches) admitting generation rescheduling and 200 million dollars (additions in $n_{2,6}, n_{2,6}, n_{2,6}, n_{3,5}, n_{4,6}, n_{4,6}$ and $n_{4,6}$ branches) when rescheduling is not an option. The system data and the topology can be obtained in (Garver 1970). Table 1 and Table 2 below show the results obtained in these simulations. 


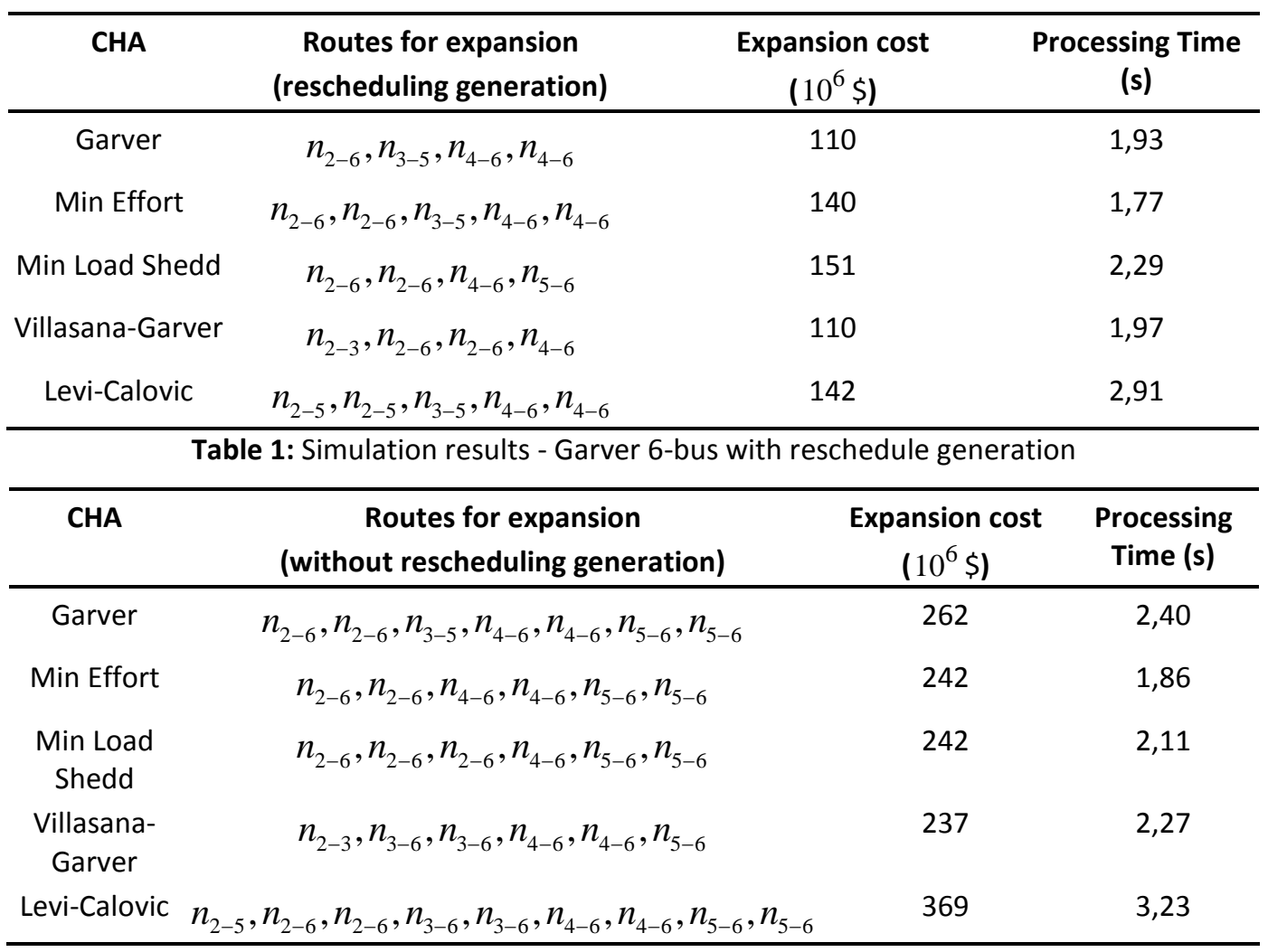

Table 2: Simulation results - Garver 6-bus without reschedule generation

As it can be seen, the solution obtained through the CHAs usually contains the optimal solutions, that is, branches $n_{3,5}$ and $n_{4,6}$ for the option with reschedule generation and branches $n_{2,6}, n_{3,5}$ and $n_{4,6}$ without this option. This feature can be used to further improve the expansion plan, for example, applying the $\mathrm{CHAs}$ only to reduce the search space and using a more powerful tool to refine the solution.

\section{Conclusions}

This paper described the main constructive heuristics algorithms used in static transmission expansion planning problems. These CHAs use different forms of modeling; Garver CHA uses the transportation model and therefore it only considers the first Kirchhoff law, the Minimum Effort and Minimum Load Shedding CHA uses the DC model, and the VillasanaGarver and the Marginal Network Levi-Calovic use an hybrid model. These efficient and simple techniques provide solutions with reasonable quality and can offer benefits in the search for the optimal expansion planning. As can be seen in the previous section, the results of the simulations confirm the hypothesis of using CHAs to reduce the search space to apply in a subsequent step a more powerful tool, generally a metaheuristic, to further refine the expansion solution, thus reducing significantly the computation effort to reach an optimal solution.

\section{References}

Bent, Russell, Carleton Coffrin, Rodrigo R. E. Gumucio, and Pascal Van Hentenryck. 2014. "Transmission Network Expansion Planning: Bridging the Gap between AC Heuristics and DC Approximations." In 2014 Power Systems Computation Conference, 1-8. IEEE. DOI: 10.1109/PSCC.2014.7038299.

Da Rocha, Manuel Costeira, and João Tomé Saraiva. 2013. “A Discrete Evolutionary PSO Based Approach to the Multiyear Transmission Expansion Planning Problem Considering 
Demand Uncertainties." International Journal of Electrical Power \& Energy Systems 45 (1): 427-42. DOI: 10.1016/j.ijepes.2012.09.009.

De Mendonça, Isabela Miranda, Ivo Chaves Silva Junior, and André L.M. Marcato. 2014. "Static Planning of the Expansion of Electrical Energy Transmission Systems Using Particle Swarm Optimization." International Journal of Electrical Power \& Energy Systems 60: 23444. DOI: 10.1016/j.ijepes.2014.02.028.

Garver, L.L. 1970. "Transmission Network Estimation Using Linear Programming." IEEE Transactions on Power Apparatus and Systems PAS-89 (7). DOI: 10.1109/TPAS.1970.292825.

Gomes, Phillipe. 2015. "Simulated Annealing with Gaussian Probability Density Function for Transmission Expansion Planning." U.Porto Journal of Engineering. http://journalengineering.fe.up.pt/article/view/104.

Gomes, Phillipe Vilaca ; Saraiva, Joao Tome; 2015. "Static Transmission Expansion Planning Using Heuristic and Metaheuristic Techniques." In PowerTech (POWERTECH), 2015. DOI: 10.1109/PTC.2015.7232377.

Levi, V.A. and Calovic, M.S. 1991. "A New Decomposition Based Method for Optimal Expansion Planning of Large Transmission Networks." \{IEEE\} Trans. Power Syst.

Monticelli, a., a. Santos, M.V.F. Pereira, S.H. Cunha, B.J. Parker, and J.C.G. Praca. 1982. "Interactive Transmission Network Planning Using a Least-Effort Criterion." IEEE Transactions on Power Apparatus and Systems PAS-101 (10): 3919-25. DOI: 10.1109/TPAS.1982.317043.

Pereira, Mario, and Leontina Pinto. 1985. "Application Of Sensitivity Analysis Of Load Supplying Capability To Interactive Transmission Expansion Planning." IEEE Transactions on Power Apparatus and Systems PAS-104 (2): 381-89. DOI: 10.1109/TPAS.1985.319053.

Rider, M.J., A.V. Garcia, and R. Romero. 2008. "Transmission System Expansion Planning by a Branch-and-Bound Algorithm." IET Generation, Transmission \& Distribution 2 (1). IET: 90. DOI: 10.1049/iet-gtd:20070090.

Romero, R., C. Rocha, J.R.S. Mantovani, and I.G. Sanchez. 2005. "Constructive Heuristic Algorithm for the DC Model in Network Transmission Expansion Planning." IEE Proceedings - Generation, Transmission and Distribution 152 (2). IET: 277. DOI: 10.1049/ip-gtd:20041196.

Villasana, R., L. Garver, and S. Salon. 1985. "Transmission Network Planning Using Linear Programming." IEEE Transactions on Power Apparatus and Systems PAS-104 (2): 349-56. DOI: 10.1109/TPAS.1985.319049.

\section{Acknowledgements}

The first author thanks CAPES Foundation, Ministry of Education of Brazil, for financing this research. 